\title{
The ENEAS Portal
}

\author{
E. Solano and J. García \\ LAEFF. P.O. Box 50727, 28080 Madrid (Spain)
}

\begin{abstract}
ENEAS (European Network of Excellence in AsteroSeismology) is formed by more than 250 scientists from $40+$ institutes. In order to optimize the communication among the ENEAS members as well as to centralize the project-related information and documentation it is necessary to build the ENEAS portal. In this paper, the main resources and services available from the portal are described.
\end{abstract}

\section{Introduction}

ENEAS has a twofold goal. It will serve as an integration and coordination mechanism of the expertise and capacities in the field of Asteroseismology across Europe. Also it will constitute the framework where young scientists will be trained and prepared to work with the ultra-high accuracy, high-temporal resolution data coming from the space missions COROT and EDDINGTON. The intrinsic nature of ENEAS makes the communication among the different participating institutes a critical issue. Networking was seen as the optimum method for the establishment of an efficient collaboration among the widely dispersed groups and the development and maintenance of a portal connecting all ENEAS activities a crucial task in this framework.

\section{The system}

The ENEAS portal is based on Postnuke, a community, collaborative, content management system. Content management systems (CMSs) are aiming at reducing development time and optimize the manpower required to manage the contents of a portal, what makes it extremely adequate for ENEAS. Postnuke allows to work within a structured environment and rapidly deliver contents such 
as articles, links, news, dynamic headlines, frequently asked questions, chat, community and file management among others. One of the big advantages of Postnuke is its modular design, which makes it possible to extend the capabilities of the system by including specific modules chosen among the great number of extra modules available. The second reason why Postnuke was chosen over similar products was the importance that the developers give to security as can be seen from the granularity of the permission set. Postnuke is based on PHP and MySql and it is a free, open-source software.

One of the potential problems of the ENEAS portal is the management of the HTML pages. In "classical" portals managed by a webmaster this problem does not exist. However, for the ENEAS portal where the information management will not be centralised in a single person but every manager will be responsible for all the activities within his/her section, the need of having some knowledge on how to handle HTML pages may constitute a major drawback. The impact of the HTML editing can be minimized by using phpWiki, an extra-module integrated into Postnuke. Wiki is a software that allows users to freely create and edit web page content using any web browser in a very easy way. Wiki supports hyperlinks and has a simple text syntax for creating new pages and crosslinks between internal pages making it ideal for the collaborative development of documentation or any other kind of content.

A second example of extra module is the Upload module, integrated in to the system to provide the ability to upload/download files (documents, images, ...) to the server.

\section{Functionalities}

The amount of information available through the ENEAS portal is given by the user's profile. Non-registered users, that is, any person accessing the ENEAS URLs (http://www.eneas.info; http://eneas.info) will have access only to limited contents: general information on the project, the list of centres participating in ENEAS, the list of Frequently Asked Questions and the ENEAS e-mail address (eneas@eneas.info). In order to have access to the full contents of the portal, it is necessary to register. The user himself can register using a login and a password. Once the registry has been completed and checked by the ENEAS portal administrator in order to guarantee the data accuracy, the user is provided with an "user profile". As of today, there are two types of profiles: the "ENEAS user" and the "ENEAS manager". Both profiles are allowed to read all the contents of the portal but the managers are also allowed to modify the contents of the sections they are responsible for. It is important to stress that the permission scheme in Postnuke is granularized what allows to create, if necesssary, any number of different profiles with different access permissions. 
The ENEAS portal as seen by a registered user is shown in Figure 1. The page is structured in three column blocks. In the left column, three sections are displayed: a) ENEAS Information, the only block available to non-registered users, b) Internal Information containing project-related documentation, the ENEAS mailing list and the tools to upload/download files, c) Areas of activity, resembling the ENEAS work package structure. From this block it is possible to have access to the ENEAS database, the data mining tools, the internet courses, software packages for the different analysis methods in a way that they can be efficiently used through the network and tools and documentation for outreach and public lecturing.

In the central column, the news of interest for the ENEAS community is posted by the responsibles of the different sections. Finally, the right column displays information on job openings at the ENEAS institutes, announcements of meetings of interest as well as ENEAS-related papers. The five most recent ones are displayed as dynamic headlines.

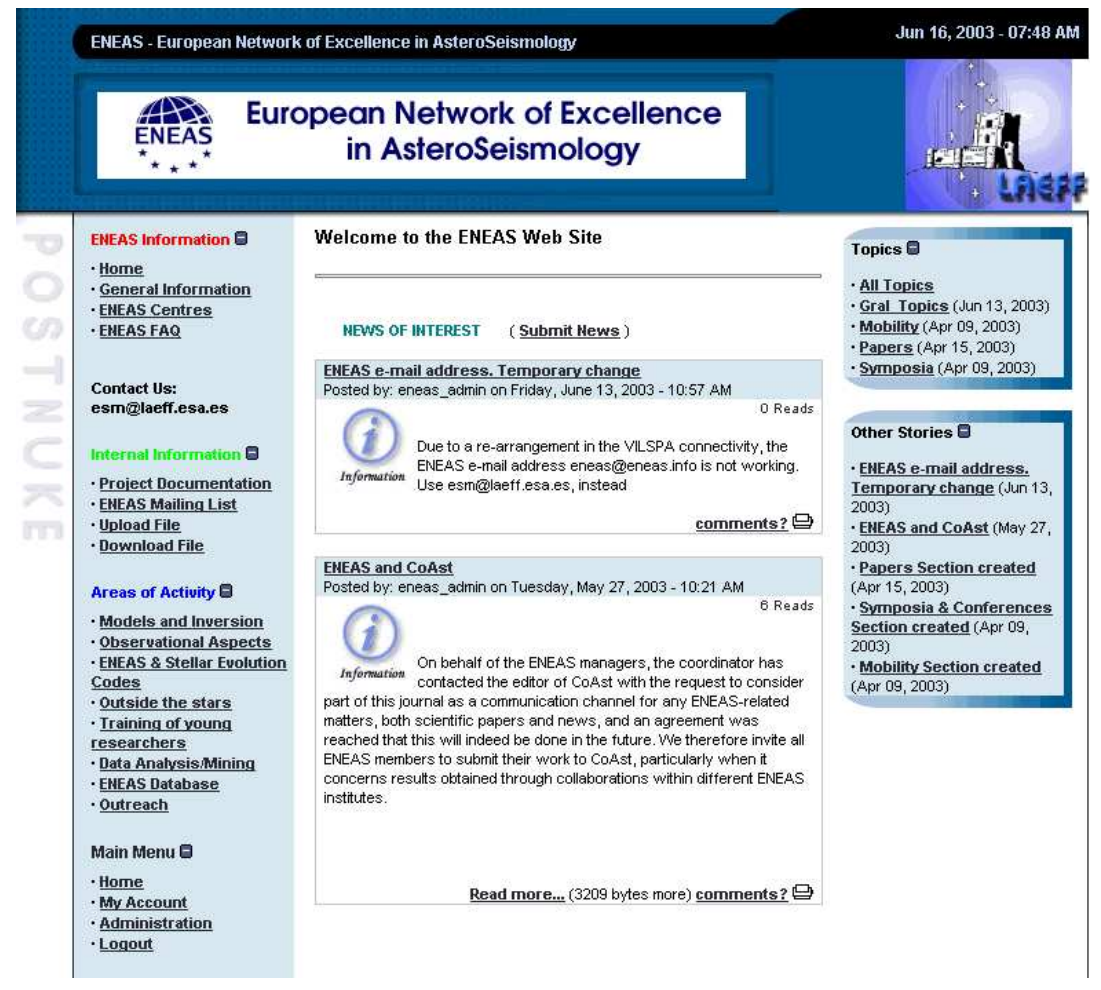

Figure 1: The ENEAS Portal 